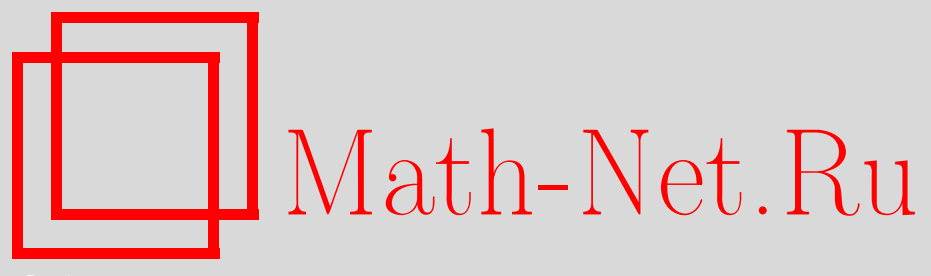

А. А. Гайфуллин, П. В. Ягодовский, Об интегрируемости $m$-значных динамик при помощи однопорожденных m-значных групп, УМH, 2007, том 62, выпуск 1, 201-202

DOI: https://doi.org/10.4213/rm5586

Использование Общероссийского математического портала Math-Net.Ru подразумевает, что вы прочитали и согласны с пользовательским соглашением http://www . mathnet.ru/rus/agreement

Параметры загрузки:

IP: 34.239 .49 .27

26 апреля 2023 г., 18:07:57

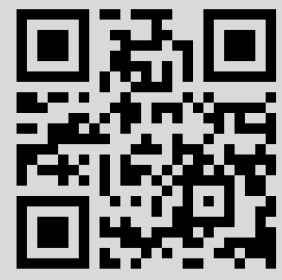




\title{
Об интегрируемости $m$-значных динамик при помощи однопорожденных $m$-значных групп
}

\author{
А. А. Гайфуллин, П. В. Ягодовский
}

В настоящей заметке доказывается достаточный признак интегрируемости многозначной динамики при помощи многозначной группы. Интегрируемость многозначной динамики понимается в смысле [1].

Пусть $S$ - конечное множество, $(S)^{m}$ - его $m$-я симметрическая степень; $m$-значной динамикой с дискретным временем на множестве $S$ называется отображение $T: S \rightarrow(S)^{m}$. Графом динамики $T$ называется граф с ориентированными ребрами на множестве вершин $S$ такой, что из вершины $s$ в вершину $s^{\prime}$ ведет столько ребер, какова кратность вхождения вершины $s^{\prime}$ в мультимножество $T(s)$. Из каждой вершины этого графа выходит ровно $m$ ребер, а число ребер, входящих в вершину $s$, равно числу прообразов элемента $s$ при динамике $T$ с учетом кратностей. Для каждого натурального числа $k$ динамика $T$ задает естественным образом кратную ей $k m$-значную динамику на том же множестве $S$.

Говорят, что множество $A$ несет на себе структуру $m$-значной группы, если заданы единица $е \in A$ и умножение $\mu: A \times A \rightarrow(A)^{m}$, удовлетворяющие естественным обобщениям аксиом ассоциативной группы. (Подробнее см. [1]-[3]. Понятие $m$-значной группы было введено В. М. Бухштабером в начале 1990-х годов.) В частности, для каждого элемента $a \in A$ должен существовать хотя бы один обратный ему элемент $b$, т.е. такой, что $e \in \mu(a, b)$ и $e \in \mu(b, a)$. Говорят, что многозначная группа порождена элементом $a_{1}$, если в степенях элемента $a_{1}$ встречаются все элементы группы; такие группы называются однопорожденными. Образующий $a_{1}$ называется эрмитовым, если он является обратным самому себе. Для любой (обычной) группы $G$ и ее подгруппы $H$ на множестве двойных смежных классов $H \backslash G / H$ существует структура многозначной бикосетной группы с умножением $\mu\left(H g_{1} H, H g_{2} H\right)=\left[H g_{1} h g_{2} H, h \in H\right]$.

Действием $m$-значной группь $A$ на множестве $S$ называется отображение $\nu: A \times S \rightarrow(S)^{m}$ такое, что мультимножества $\nu\left(a_{1}, \nu\left(a_{2}, s\right)\right)$ и $\nu\left(\mu\left(a_{1}, a_{2}\right), s\right)$ совпадают для любых $a_{1}, a_{2} \in A, s \in S$ и $\nu(e, s)=[s, s, \ldots, s]$. Согласно [1], динамика $T$ на множестве $S$ называется интегрируемой при помощи однопорожденной многозначной группь $A$ с образующим $a_{1}$, если существует действие $\nu$ группы $A$ на $S$ такое, что мультимножества $T(s)$ и $\nu\left(a_{1}, s\right)$ совпадают для любого $s \in S$.

В [4] для широкого класса многозначных динамик, а именно, для динамик, задаваемых однородными графами (см. ниже), было доказано, что некоторые кратные им динамики интегрируемы, и поставлена задача о характеризации динамик, интегрируемых без перехода к кратным. В настоящей статье мы решаем эту задачу, доказывая достаточный признак интегрируемости многозначных динамик, который, в частности, обеспечивает интегрируемость всех динамик, задаваемых однородными графами. Доказательство основано на конструкции, являющейся обобщением конструкции работы [4].

ТЕОРема 1 (достаточный признак интегрируемости). Пусть $m$-значнал динамика T на множестве $S$ такова, что каждый элемент $s \in S$ имеет с учетом кратностей ровно $m$ прообразов. Тогда динамика $T$ интегрируема при помощи некоторой бикосетной однопорожденной $m$-значной группы.

ДокАзАтельство. Рассмотрим граф динамики $T$; пусть $E$ - множество его ребер. Для каждой вершины $s$ обозначим через $E_{s,-}$ множество ребер, выходящих из вершины $s$, и через $E_{s,+}$ множество ребер, входящих в вершину $s$. Тогда $\left|E_{s,-}\right|=\left|E_{s,+}\right|=m$. Пусть $\gamma$ - множество из $m$ элементов, $H$ - какая-либо транзитивная группа перестановок его элементов. Для каждой вершины $s$ выберем произвольным образом картирующие биекции $\psi_{s, \pm}: \gamma \rightarrow E_{s, \pm}$. Биекции $\psi_{s,-}$ позволяют определить действие 
группы $H$ на множестве $E$ : если $\varepsilon \in E_{s,-}$, то $h(\varepsilon)=\psi_{s,-}\left(h\left(\psi_{s,-}^{-1}(\varepsilon)\right)\right)$. Таким образом, можно считать группу $H$ подгруппой группы перестановок множества $E$. Отображение, ставящее в соответствие каждому ребру его начало, задает отождествление $E / H=S$. Положим $t(\varepsilon)=\psi_{s,-}\left(\psi_{s,+}^{-1}(\varepsilon)\right)$, если $\varepsilon \in E_{s,+}$. Тогда $t-$ перестановка множества $E$. Обозначим через $G$ подгруппу группы перестановок множества $E$, порожденную подгруппой $H$ и перестановкой $t$. Многозначная бикосетная группа $A=H \backslash G / H$ естественно действует на множестве $S=E / H$, причем динамика, задаваемая ее образующим $H t H$, кратна динамике $T$ с коэффициентом $\frac{|H|}{m}$. Группа $A$ будет интегрировать динамику $T$, например, если в качестве $H$ взять группу $\mathbb{Z}_{m}$, действующую на множестве $\gamma$ циклическими перестановками. Теорема 1 доказана.

Пусть Г - обобщенный однородный граф, т.е. граф с неориентированными (возможно, кратными) ребрами и ориентированными (возможно, кратными) петлями, все вершины которого имеют степень $m$. Определим на множестве его вершин динамику $T$, переводящую вершину в мультимножество ее соседей. Граф этой динамики может быть получен из Г заменой каждого ребра на пару ребер, имеющих противоположные ориентации. Определим картирующие биекции так, чтобы перестановка $t$ меняла местами ребра каждой такой пары, а петли оставляла неподвижными. Тогда $t^{2}=1$.

СлЕДСтвиЕ 1. Естественная многозначная динамика на множестве вершин обобщенного однородного графа интегрируема при помощи некоторой бикосетной однопорожденной многозначной группы с эрмитовым образующим.

Интегрируемость динамики, кратной динамике $T$, в случае, когда $\Gamma$ - симметрический граф без петель и кратных ребер, была доказана в серии работ [5], [2], [3]. Интегрирующая группа имела вид $H_{\Gamma} \backslash G_{\Gamma} / H_{\Gamma}$, где $G_{\Gamma}$ - группа автоморфизмов графа $\Gamma$, действующая транзитивно на его вершинах и его ребрах, $H_{\Gamma} \subset G_{\Gamma}$ - стабилизатор некоторой вершины. Конструкция из [5], [2], [3] и конструкция из настоящей заметки приводят, вообще говоря, к различным многозначным группам, поскольку введение картирующих биекций нарушает симметрию графа. Подчеркнем, что построенная в доказательстве теоремы 1 группа $A$ зависит не только от выбора группы $H$, но и от выбора картирующих биекций. Например, для полного графа на 4 вершинах при $H=\mathbb{Z}_{3}$ существует ровно 3 принципиально различных способа выбора картирующих биекций. В результате мы получаем трехзначные группы, интегрирующие соответствующую динамику, порядков 2, 12 и 38 . Первая из этих групп может быть построена и с помощью конструкции из работ [5], [2], [3].

В завершение авторы хотят выразить свою глубокую благодарность профессору В. М. Бухштаберу за помощь и поддержку в работе.

\section{Список литературы}

[1] V. Buchstaber, Mosc. Math. J., 6:1 (2006), 57-84. [2] П. В. Ягодовский, Записки науч. сем. ПОМИ, 292 (2002), 161-174. [3] П. В. Ягодовский, Записки науч. сем. ПОМИ, 325 (2005), 225-242. [4] В. М. Бухштабер, А. А. Гайфуллин, УМН, 61:3 (2006), 171-172. [5] П. В. Ягодовский, УМН, 57:1 (2002), 143-144.

\section{А. А. Гайфуллин (А. A. Gaifullin)}

Московский государственный университет им. М. В. Ломоносова

E-mail: gaifull@mccme.ru

\section{П. В. Ягодовский (Р. V. Yagodovskii)}

Московский государственный университет им. М. В. Ломоносова,

Финансовая академия при Правительстве РФ

E-mail: korcsak@list.ru
Представлено В. М. Бухштабером Принято редколлегией 17.11.2006 